\title{
Equalization of Linearly Frequency-Selective Fading Channels
}

\author{
Wing Seng Leon, Member, IEEE, Umberto Mengali, Fellow, IEEE, \\ and Desmond P. Taylor, Fellow, IEEE
}

\begin{abstract}
A simple technique for removing intersymbol interference (ISI) introduced by "linearly frequency-selective" fading channels is presented. The technique involves the optimization of the overall impulse response of the transmit and receive filters and effectively reduces the channel to one which is flat fading. Computer simulation results show that this equalization method works for channels with small delay spreads.
\end{abstract}

Index Terms - Channel models, equalization, fading, frequency-selective.

\section{INTRODUCTION}

A LTHOUGH the maximum-likelihood receivers proposed in [1] and [2] are designed for a general Rayleigh frequency-selective fading channel, complexity issues make their implementation difficult. Furthermore, these receivers require prior knowledge of the second-order channel statistics. Therefore, it is advantageous to design simple receivers for a more limited range of channels.

Here, we develop a new approach for the reception of signals transmitted over a channel which is slowly fading and exhibits small delay spread. Following [3] and [4], the channel is assumed to be sufficiently modeled as a two-term Taylor's series expansion; it is then linearly frequency-selective [3]. The proposed approach uses transmit and receive filters which effectively remove the intersymbol interference (ISI) induced by the channel. The response of the filters is designed by minimizing the mean square error (MSE) between the received signal and the desired (ISI-free) signal. Hence, a receiver with limited complexity such as those discussed in [5]-[7] for a time-selective channel could be used to detect the transmitted signals.

Section II defines the channel and signal models. The optimal overall response of the transmit and receive filters is developed in Section III. In Section IV, bit-error rate (BER) results from computer simulation of a communication system using this equalization approach and a predictor receiver similar to the one in [6] are presented. Some concluding remarks are given in Section V.

\section{Channel And Signal Model}

The transmitted complex baseband signal is written as

$$
s(t)=\sum_{i=-\infty}^{\infty} a_{i} g(t-i T)
$$

Paper approved by J. Chuang, the Editor for Wireless Networks of the IEEE Communications Society. Manuscript received August 26, 1996; revised February 24, 1997 and June 13, 1997.

W. S. Leon and D. P. Taylor are with the Department of Electrical and Electronic Engineering, University of Canterbury, Christchurch, New Zealand (e-mail: leonws@elec.canterbury.ac.nz; taylor@elec.canterbury.ac.nz).

U. Mengali is with the Department of Information Engineering, University of Pisa, 56126 Pisa, Italy (e-mail: mengali@pimac2.iet.unipi.it). where $g(t)$ is the impulse response of the transmit filter, $1 / T$ is the symbol rate, and $\left\{a_{i}\right\}$ are uncorrelated data symbols satisfying the relation

$$
E\left[a_{i} a_{j}^{*}\right]= \begin{cases}1, & \text { for } i=j \\ 0, & \text { otherwise }\end{cases}
$$

where the superscript $*$ denotes complex conjugate. The signal in (1) is transmitted over a Rayleigh-fading frequencyselective channel. We assume that the channel is slowly fading such that its impulse response does not vary significantly over a symbol period. We also assume that only linear channel variations are significant. Following [3] and [4], the signal component of the channel output is then expanded as a Taylor's series truncated to the first two terms and is written as $r(t)=\sum_{i=-\infty}^{\infty} T_{0}(i) a_{i} g(t-i T)+\sum_{i=-\infty}^{\infty} T_{1}(i) a_{i} g^{\prime}(t-i T)$.

$T_{0}(i)$ and $T_{1}(i)$ are correlated complex Gaussian random variables known as the time-selective coefficients. They are related to the complex random gains of each of the delay paths of the channel. As in [4], we let the mean delay path be zero and, hence, the cross-correlation of the time-selective coefficients becomes

$$
E\left[T_{0}(i) T_{1}^{*}(i)\right]=0 .
$$

The term $g^{\prime}(t)$ in (3) represents the time derivative of the transmit filter impulse response $g(t)$.

The channel output $r(t)$ is filtered by the receive filter $w(t)$. Thus, the overall response of the transmit and receive filters is

$$
p(t)=g(t) \otimes w(t)
$$

where $\otimes$ denotes convolution. It can also be shown that the derivative of $p(t)$ is given by

$$
p^{\prime}(t)=g^{\prime}(t) \otimes w(t) .
$$

The output of the receive filter $y(t)$ is sampled at time $t=k T$ and the resulting sample is

$y(k)=\sum_{i=-\infty}^{\infty} T_{0}(i) a_{i} p[(k-i) T]+\sum_{i=-\infty}^{\infty} T_{1}(i) a_{i} p^{\prime}[(k-i) T]$.

\section{Channel EQualization}

It is reasonable to choose the transmit filter $g(t)$ and receive filter $w(t)$ so that the overall response $p(t)$ satisfies the Nyquist criterion. Therefore, only the second term of (7) contributes any ISI to the sampled output $y(k)$. If ISI is completely removed, the received sample is reduced to

$$
y_{\text {ideal }}(k)=T_{0}(k) a_{k} .
$$


The expression in (8) is equivalent to a received sample from a flat-fading channel. The error between the actual received sample (7) and the ideal ISI-free sample (8) is

$$
\begin{aligned}
e(k)=T_{0}(k)[1-p(0)] a_{k}- & \sum_{i \neq k} T_{0}(i) a_{i} p[(k-i) T] \\
& -\sum_{i} T_{1}(i) a_{i} p^{\prime}[(k-i) T]
\end{aligned}
$$

We are now motivated to optimize $p(t)$ such that the MSE between the ideal and actual received sample is minimized. Let the MSE be

$$
J=E\left[|e(k)|^{2}\right]
$$

Using (2), (4), and (9), (10) is rewritten as

$$
J=A_{0}[1-p(0)]^{2}+A_{0} \sum_{k \neq 0} p^{2}(k T)+A_{1} \sum_{k} p^{2}(k T)
$$

where $A_{0}=E\left[\left|T_{0}(k)\right|^{2}\right]$ and $A_{1}=E\left[\left|T_{1}(k)\right|^{2}\right]$. Letting $\gamma=A_{1} / A_{0}$, (11) may be rewritten as

$$
J=A_{0}\left[1+\sum_{k} p^{2}(k T)+\gamma \sum_{k} p^{2}(k T)-2 p(0)\right] .
$$

By inspection of (12), minimizing $J$ amounts to minimizing

$$
J^{\prime}=1+\sum_{k} p^{2}(k T)+\gamma \sum_{k} p^{\prime 2}(k T)-2 p(0) .
$$

To do this, we rewrite the second, third, and fourth term on the right-hand side of (13) by using the Fourier transform, Poisson's summation formula, and the frequency convolution theorem [8]. Neglecting details, (13) becomes

$$
\begin{aligned}
J^{\prime}= & \frac{1}{T} \sum_{k} \int_{-\infty}^{\infty} P(f) P\left(\frac{k}{T}-f\right) d f \\
& -\frac{\alpha}{T} \sum_{k} \int_{-\infty}^{\infty}\left(\frac{k}{T}-f\right) f P(f) P\left(\frac{k}{T}-f\right) d f \\
& -2 \int_{-\infty}^{\infty} P(f) d f
\end{aligned}
$$

where $\alpha=4 \pi^{2} \gamma, f$ is the frequency variable, and $P(f)$ is the Fourier transform of $p(t)$. In order to simplify the optimization and ensure that the solution is realizable, $P(f)$ is assumed to be real valued and bandlimited to $|f| \leq 1 / T$, i.e., $P(f)=0$ for $|f| \leq 1 / T$. The minimization of $J^{\prime}$ and the optimum $P(f)$ and $p(t)$ are obtained by using calculus of variations techniques. Again omitting details, the optimum overall frequency response of the transmit and receive filter is found to be

$$
p^{\circ}(f)=T(1-|f| T)
$$

which corresponds to an impulse response

$$
p^{o}(t)=\operatorname{sinc}^{2}\left(\frac{t}{T}\right)
$$

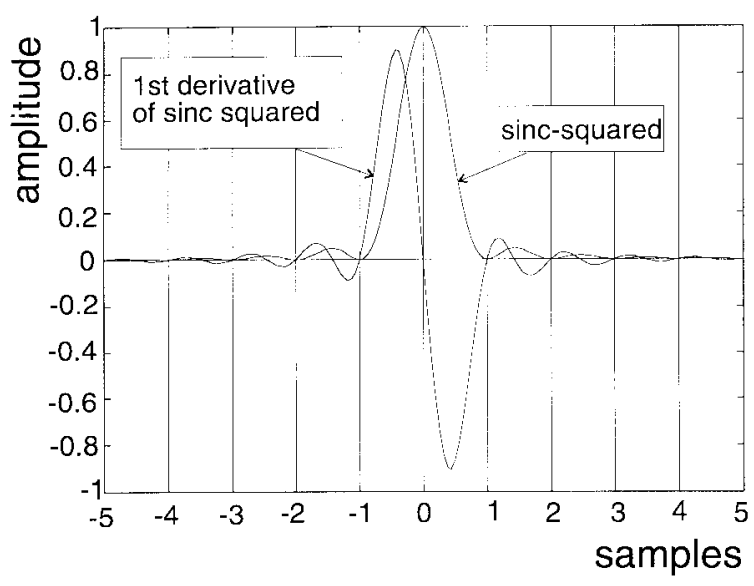

Fig. 1. Sinc-squared impulse response and its first derivative.

The impulse response $p^{\circ}(t)$ and its first derivative $p^{o^{\prime}}(t)$ are shown in Fig. 1. Note that $p^{\circ}(t)$ satisfies the Nyquist criterion for zero ISI.

It is seen in (15) and (16) that the optimum overall response is independent of the ratio $\gamma$ which is previously defined. Since $p^{\circ}(t)$ is Nyquist, the first and second terms of (11) are zero. It can also be shown that the summation in the third term is

$$
\sum_{k} p^{2}(k T)=0
$$

for $p(k T)=p^{\circ}(k T)$. This implies that the total MSE in (11) is zero and the received samples are ISI-free. Since ISI is completely removed, the channel is equalized and effectively behaves like a flat-fading channel.

\section{Simulation Results}

Using the optimal filter, we simulated a system to evaluate its performance. The derivation in Section III assumed a noiseless environment. However, additive noise is always present at the front end of the receiver. In the computer simulation, white Gaussian noise with power spectral density of $N_{\circ}$ is added to the output of the channel. Since the channel is assumed to be "linearly frequency-selective," it would be necessary to know the types of channel for which this assumption is true. This is dependent on the delay spread of the channel [4]. In the simulation, channels with a uniform delay profile and maximum delay spreads ranging from $0.05 T$ to $0.2 T$ were used. A three-ray wide-sense stationary uncorrelated scattering channel with normalized fade rate (time-bandwidth product) of $B T=0.01$ was used.

A predictor sequence estimator similar to the one described in [6] which is designed for flat-fading channels has been used in the simulations. The receiver was made adaptable to different fade rates by using the recursive least squares algorithm to update the predictor taps [9] with baud rate sampling and processing. A predictor with ten taps was used. Since we are interested in the steady-state performance of the receiver, the predictor was trained for 10000 symbols to ensure that the tap weights converge. A 16-state Viterbi algorithm with a decision delay of 30 symbols was used. The modulation scheme used is quadrature phase-shift keying (QPSK) with a 


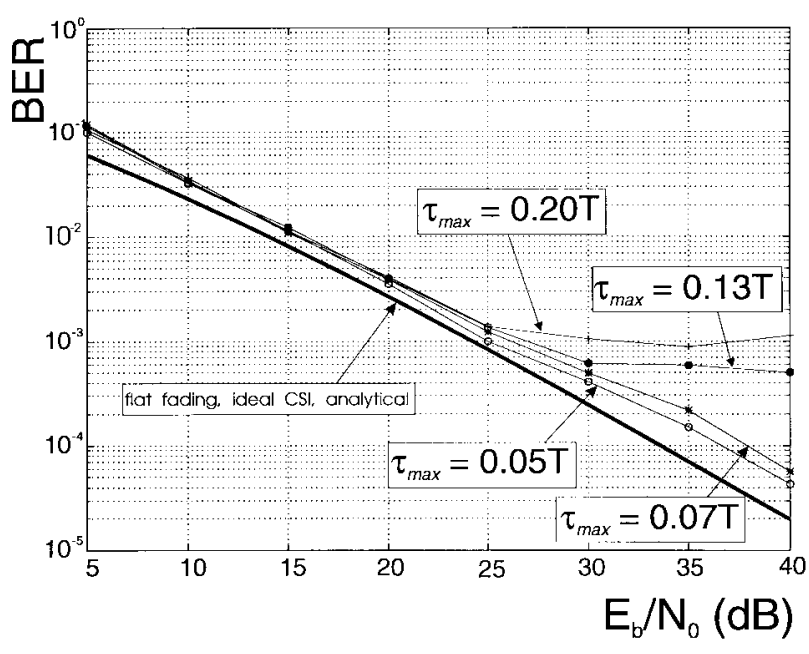

Fig. 2. Simulated BER curves for a predictor receiver for a linearly frequency-selective fading channel. The analytical BER curve using ideal CSI is in bold. The optimum sinc-squared pulse shape is used.

reference symbol inserted every ten data symbols to eliminate any phase ambiguity. This is analogous to a system with differential encoding and decoding. The system was chosen to clearly isolate the effects of the equalizing pulse shape. The average received bit energy is $E_{b}$. For convenience, all filtering was performed at the transmitter using a filter with impulse response similar to (16). It is effectively a predistortion filter which equalizes the channel. This will incur at most a 3-dB loss in average $E_{b} / N_{o}$ compared to matched filtering.

The results of simulation are shown in Fig. 2. For channels with maximum delay spread of $\tau_{\max }=0.05 T$ and $0.07 T$, the BER curves do not exhibit any error floors. However, at higher signal-to-noise ratio (SNR), the performance for the channel with $\tau_{\max }=0.07 T$ is about $2-3 \mathrm{~dB}$ worse than for the $\tau_{\max }=0.05 T$ channel. For comparison, we have included an analytic BER curve using ideal channel state information (CSI) following the method in [10]. The simulation results agree quite closely with the analytic BER curve at low SNR as shown. However, for channels with $\tau_{\max }=0.13 T$ and $0.2 T$, the corresponding BER curves began to floor at SNR's of about 30 and $25 \mathrm{~dB}$, respectively. As shown in [4], the error between the conventional tapped-delay line model and the power series model increases with increasing delay spread. Furthermore, the modeling error increases with decreasing order of the power series. Therefore, the assumption of a linearly frequency-selective channel or a two-term $f$-power series does not hold true for the channels with higher delay spreads. The irreducible error floor in such a channel is present because the difference between the actual channel impulse response and the linearly frequency-selective model becomes significant.

\section{CONCLUSION}

It has been shown that the optimum overall impulse response of the transmit and receive filter is sinc-squared for a linearly frequency-selective fading channel with small delay spread. By using this overall response, the filtered received samples are equalized and the channel is effectively reduced to a flat-fading channel. Simulations have shown that the linearly frequency-selective fading channel is indeed equalized and behaves like a flat-fading channel by using this approach. A receiver designed for flat fading can then be used for the reception of signals transmitted over such a channel. However, if the maximum delay spread of the channel increases, modeling error between the actual channel impulse response and the two-term $f$-power series model also increases and the simple approach fails. The major benefit of using this approach is that it can be simply implemented using a time-invariant filter. Its main disadvantage is that the filter impulse response is one with $100 \%$ rolloff and therefore requires a larger transmission bandwidth.

\section{REFERENCES}

[1] Q. Dai and E. Shwedyk, "Detection of bandlimited signals over frequency selective Rayleigh fading channels," IEEE Trans. Commun., vol. 42, pp. 941-950, Feb./Mar./Apr. 1994

[2] X. Yu and S. Pasupathy, "Innovation-based MLSE for Rayleigh fading channels," IEEE Trans. Commun., vol. 43, pp. 1534-1544, Feb./Mar./Apr. 1995

[3] P. A. Bello, "Characterization of randomly time-variant linear channels," IEEE Trans. Commun. Syst., vol. 11, pp. 360-393, Dec. 1963.

[4] G. Deng, J. Cavers, and P. Ho, "A reduced dimensionality propagation model for frequency selective Rayleigh fading channels," in ICC, Seattle, WA, 1995, pp. 1158-1162.

[5] P. Y. Kam and C. H. Teh, "An adaptive receiver with memory for slowly fading channels," IEEE Trans. Commun., vol. COM-32, pp. 654-659, June 1984.

[6] G. M. Vitetta and D. P. Taylor, "Maximum likelihood decoding of uncoded and coded PSK signal sequences transmitted over Rayleigh frequency-flat fading channels," IEEE Trans. Commun., vol. 43, pp. 2750-2758, Nov. 1995 .

[7] P. K. Varshney and A. H. Haddad, "A receiver with memory for fading channels," IEEE Trans. Commun., vol. COM-26, pp. 278-283, Feb. 1978.

[8] A. Papoulis, The Fourier Integral and Its Application. New York: McGraw-Hill, 1962.

[9] S. Haykin, Adaptive Filter Theory, 2nd ed. Englewood Cliffs, NJ: Prentice-Hall, 1991.

[10] B. Hart and D. P. Taylor, "Extended MLSE diversity receiver for the time and frequency selective channel," in ICC, Dallas, TX, 1996, pp. 874-878. 